\title{
Disease Related Malnutrition in Hospital Outpatients, - Time for Action
}

\author{
Holst Mette $^{1^{*}}$, Zacher Nina ${ }^{2}$, Østergaard Trine ${ }^{2}$, Mikkelsen Sabina ${ }^{2}$ \\ ${ }^{1}$ Center for Nutrition and Bowel Disease, Aalborg University Hospital and Clinical Institute, Aalborg University, \\ ${ }^{2}$ Department of Health Science and Technology, Aalborg University, Denmark
}

\begin{abstract}
Background: Malnutrition is common in hospitalized patients, and is known to have negative consequences on the course and outcome of treatment. As the proportion and burden of disease is increasing in the outpatient setting, a focus on malnutrition in the overall outpatient setting may be required.

Aim: This study aimed to investigate the prevalence of unintended weight loss and associated risk factors in outpatients visiting five different specialties.

Methods: In this cross sectional study, data regarding weight loss during the past three months, "eaten less" the past week and associated risk factors, was collected consecutively through a one-page questionnaire, for a full week in each of five ambulatories at Aalborg University Hospital, Denmark. Descriptive analysis, $\mathrm{Chi}^{2}$-analysis and multiple logistic regression analysis were used for statistical analysis.

Results: Of the 713 participants, 26\% had an unintended weight loss within the last three months and $21 \%$ had eaten less than usual over the past week. Patients $>80$ years [CI95\%: 1.496;4.358] and underweight patients [CI95\%: 1.766;8.884] had significant increased odds for 'unintended weight loss', while women [CI 95\%: 1.216;2.542], patients from the department of medical gastroenterology [CI95\%:1.499;4.205], department of gastrointestinal surgery [CI95\%: 1.289;3.797] and department of lung medicine [CI95\%: $1.353 ; 4.129$ ] as well as underweight patients [CI95\% 1.202;4.742] showed increased odds for 'eaten less'.

Conclusion: Based on the statistical analysis, there is a high risk of unintended weight loss in outpatient clinics. Therefore, guidelines for handling disease related under nutrition in outpatient settings are required.

Keywords: Malnutrition, Outpatient, BMI, Weight loss.
\end{abstract}

\section{Introduction}

Malnutrition in hospitalized patients has been widely described and is known to have significant consequences for the course and outcome of treatment. Thus, a significant amount of research has underlined disease related malnutrition, as being associated with increased risk of infections, complications, need of prolonged care and decreased quality of life, as well as mortality [1-7].

Malnutrition is defined as a consequence of inadequate intake of proteins and energy, which, in combination with increased requirements due to the stressed metabolism, can lead to a reduction of appetite and less food intake, forming a negative energy balance, leading to weight loss [8].

In a health economic perspective, disease related malnutrition has been shown to account for $10 \%$ of total public health expenditure. According to a Danish study, the additional costs for medical patients due to malnutrition in 2008 was
1.726 DKK per 3.4 extra hospital days in 28.700 admissions summing up to DKK 168 million, which may be saved through better and earlier nutritional efforts $[9,10]$. Early detection of malnutrition including sarcopenia, thus preventing cachexia, has been advocated for the possibility to recover $[6,11,12,13]$.

Corresponding Author: Mette Holst, Center for Nutrition and Bowel Disease, Aalborg University Hospital and Clinical Institute, Aalborg University, Mølleparkvej 4, Reception 3, 9000 Aalborg, Denmark E-mail: mette.holst@rn.dk Received date: March 25, 2019; Accepted date: April 02, 2019; Published date: April 03, 2019.

DOI: https://doi.org/10.31546/IJFSNR.1002 
Several studies have highlighted that early nutrition intervention, may have a beneficial effect on avoiding unplanned weight loss, reducing risks of complications and improving, quality of life maintenance and functional level [14-20].In Denmark there is a significant trend towards increasing the frequency of ambulatory care for patients compared to in-hospital treatment, where number of total inhospital days has decreased every year for the past decade (Denmarks Statistics http://www.statistikbanken.dk/). These care strategies include treatment of chronic diseases as well as acute diseases and different types of cancer. Furthermore, the increasing age population, has increased the number of ambulatory follow ups and diagnostic investigations (The Danish National Research and Analysis Center for Welfare, Sept. 2018).

While The National Board of Health has national recommendations for nutritional efforts in hospital as well as in community dwelling elderly, there are no recommendations for ambulatory care [21]. Due to the development in population composition and treatment strategies, patients in the outpatient clinic may also be at risk of malnutrition, which may affect the course and outcome of treatment. Only a few studies outside Denmark have looked into the risk of malnutrition in hospital outpatients. These have found a sound variation of patients at risk, depending of diagnose, setting and screening method used. A study from UK found a prevalence of $21.3 \%$ at risk of malnutrition in a Gastroenterology Clinic [22], while an Australian study found a prevalence of $17 \%$ malnourished patients and $37.2 \%$ at risk of malnutrition in ambulatory cancer patients [15]. Furthermore, a study from Spain found a great variation of malnutrition risk within different cancer diagnosis [23].

In the present study, we aimed to investigate the prevalence of unintentional weight loss as a primary marker for the risk of disease related malnutrition but including also patient assessment of "eaten less" within one week and body mass index (BMI), in five different outpatient clinics at Aalborg University Hospital, Denmark [24].

\section{Methods}

This study was designed as a questionnaire based cross sectional investigation including all patients visiting five different outpatient clinics for each one week; Gastroenterology, Infectious Medicine, Pulmonary Medicine, Abdominal Surgery and Hematology.

\section{Questionnaire:}

The questionnaire included nine questions of 1.Gender, 2.Age, 3.Reason for visit (treatment, investigation, ambulatory follow-up, results of physical examination, course of investigation), 4.Unintentional weight loss within the past three months, 5.Eaten less or as normal within the past week,
6.Nutritional impact symptoms, 7.Lack of help for cooking / eating alone, 8.Talked to professionals about weight loss, 9.Intentional weight loss.

\section{Inclusion}

Roll up boards including the text "Ambulatory nutrition week" including information about the study, were placed at the entrance and reception of the outpatient clinics. After registering at the receptive outpatient clinics patients were approached by the investigators, giving information and inviting them to participate in the investigation. A weighing scale and height measuring scale were placed close to the reception. If willing to participate, patients could fill in the questionnaire themselves, or have the questions read and filled in by the investigators. Patients were advised weighing, if they did not weigh themselves on the particular morning. Likewise, if they had not had their height measured recently.

Data were entered in EPIDATA 4.2 and the entrance was double checked. Missing data were entered as 999 and excluded from analysis.

\section{Statistics}

A descriptive analysis was made and presented as number $(\mathrm{N})$ and mean $\pm \mathrm{SD}$. Chi2 and multiple logistic regression analysis were used to test for association between "reason for visit" and "unintended weight loss" as well for association between "reason for visit" and "eaten less". $\mathrm{P}<0.05$ was considered significant and Odds Ratio (OR) was given with a 95\% confidence interval (CI). The associations were adjusted for gender, departments, BMI and age. Data were analyzed using SAS for Windows version 9.4.

\section{Ethical considerations}

Participation was voluntary and no information gathered could lead back to the particular patient. The regional ethic committee was approached about the study, and found no reason for full application. Application ID: 2018-146.

\section{Results}

The study included 713 patients in total and the highest prevalence of outpatients came from the hematology department $(\mathrm{N}=219)$. Of these, $52 \%$ were male and mean age was 59.8 years. Mean BMI was $26.14 \mathrm{~kg} / \mathrm{m} 2$ and the highest prevalence for reason for visit were investigation ( $\mathrm{N}=249)$. Of the outpatients, $25.6 \%$ had an unintentional weight loss within the past three months and mean weight loss was $7.5 \mathrm{~kg}$. $21 \%$ of the patients 
Mette Holst et al.

have eaten less within the past week. The highest prevalence of nutritional impact symptoms among outpatients who had eaten less within the past week was no appetite $(\mathrm{N}=108)$.
Finally, $7.2 \%$ of the sample had an intentional weight loss Table-1 shows demographic information.

\begin{tabular}{|l|l|}
\hline Variable & $\mathbf{N}=$ \\
\hline $\begin{array}{l}\text { Outpatient clinic: } \\
\text { Gastroenterology (M) }\end{array}$ & 170 \\
$\quad$ Infektious Medicine (I) & 24 \\
$\quad$ Abdominal Surgery (A) & 176 \\
$\quad$ Pulmonary Medicine (L) & 219 \\
$\quad$ Total & 124 \\
\hline Sex (Male) & 713 \\
\hline BMI, kg/m ${ }^{2}$ & $52 \%$ \\
\hline Age, years & 26.14 (SD 6) \\
\hline Unintentional weight loss, Yes \% & 59.8 (SD 17.3) \\
\hline Unintentional weight loss $>3$ months, KG & $25.6 \%$ \\
\hline Eaten less than usual the past week, yes \% & 7.5 (SD 5.6) \\
\hline Intentional weight loss, yes \% & $21 \%$ \\
\hline Reason for visit: & $7.2 \%$ \\
Treatment & \\
Investigation & 196 \\
Ambulatory follow-up & 249 \\
Results of physical examination & 217 \\
Course of investigation & 60 \\
\hline Nutritional impact symptoms: & 47 \\
Nausea & Pain \\
Worries & 52 \\
Swallowing problems & 57 \\
No appetite & 25 \\
\hline & 16 \\
\hline
\end{tabular}

Table 1: Demographic information

The association between "unintended weight loss" and different variables are presented in table 2. There was an association between "unintended weight loss" and age $(p<0.05)$, and the oldest age group had a significant higher risk of having an unintended weight loss (OR=2.554; CI (1.496-4.358)). An association was found between "unintended weight loss" and BMI $(p<0.05)$, as the underweight patients had a significant higher risk of having an unintended weight loss (OR=3.960; CI (1.766-8.884)).

Finally, there was an association between "unintended weight loss" and the reason for visit $(\mathrm{p}<0.05)$, as outpatients whose reason for visit were ambulatory follow-up, had a significant lower risk of having an unintended weight loss. There were no association between "unintended weight loss" and sex or outpatient clinic ( $p>0.05)$. The association between "eaten less" and different variables are presented in table 3. There was an association between "eaten less" and sex ( $\mathrm{p}<0.05)$, and women had a significant higher risk of having eaten less $(\mathrm{OR}=1.758$ CI (1.216-2.542)). 


\begin{tabular}{|c|c|c|c|}
\hline Variable & p-value & Odds ratio & Confidence interval \\
\hline Sex (Women): & 0.5443 & 1.1102 & $0.790-1.560$ \\
\hline Outpatient clinic: & 0.8913 & & \\
\hline Gastroenterology (M) & & 1.1650 & $0.734-1.849$ \\
\hline Infectious Medicine (I) & & 1.0710 & $0.404-2.838$ \\
\hline Abdominal Surgery (A) & & 1.1650 & $0.734-1.849$ \\
\hline Pulmonary Medicine (L) & & 1.2920 & $0.783-2.132$ \\
\hline Age: & $0.0015^{*}$ & & \\
\hline 0-19 & & 0.984 & $0.309-3.134$ \\
\hline $20-30$ & & 0.969 & $0.558-1.682$ \\
\hline $40-59$ & & 0.785 & $0.515-1.196$ \\
\hline $80-99$ & & $2.554^{*}$ & $1.496-4.358$ \\
\hline BMI**: & $0.0020^{*}$ & & \\
\hline Underweight & & $3.960 *$ & $1.766-8.884$ \\
\hline Overweight & & 0.876 & $0.590-1.299$ \\
\hline Obesity class 1 & & 0.692 & $0.378-1.265$ \\
\hline Obesity class 2 & & 0.592 & $0.218-1.608$ \\
\hline Obesity class 3 & & 0.583 & $0.164-2.082$ \\
\hline Reason for visit: & $0.0495^{*}$ & & \\
\hline Treatment & & $0.831 * * *$ & $0.534-1.294$ \\
\hline Ambulatory follow-up & & $0.439 * / * * *$ & $0.439-0,710$ \\
\hline Results of physical examination & & $0.748 * * *$ & $0.370-1.512$ \\
\hline Course of investigation & & $0.915 * * *$ & $0.405-2.065$ \\
\hline
\end{tabular}

Table 2: The association between "unintentional weight loss" sex, outpatient clinic, age and BMI. Men, hematology department, 60-79 years, normal weight and investigation are the reference group. $* \mathrm{p} \leq 0.05$ level of significance ** BMI is classified according to the WHO classification of BMI groups *** Adjusted for sex, BMI, department and age.

There was an association between "eaten less" and outpatient clinic ( $\mathrm{p}<0.05)$, and the departments $\mathrm{M}, \mathrm{A}$ and $\mathrm{L}$ had outpatients who had a significant higher risk of having eaten less (M: OR=2.511; CI (1.499-4.205) A: OR=2.212; CI (1.2 89-3.797) L: OR=2.363; CI (1.353-4.129)).
Finally, there was an association between "eaten less" and the reason for visit $(\mathrm{p}<0.05)$, and outpatients, whose reason for visit were ambulatory follow-up and treatment, had a significant lower risk of having eaten less. 


\begin{tabular}{|c|c|c|c|}
\hline Variable & p-value & Odds ratio & Confidence interval \\
\hline Sex (Women): & $0.0026^{*}$ & $1.758^{*}$ & $1.216-2.542$ \\
\hline Outpatient clinic: & $0.0033^{*}$ & & \\
\hline Gastroenterology (M) & & $2.511^{*}$ & $1.499-4.205$ \\
\hline Infectious Medicine (I) & & 1.303 & $0.416-4.086$ \\
\hline Abdominal Surgery (A) & & $2.212 *$ & $1.289-3.797$ \\
\hline Pulmonary Medicine (L) & & $2.363^{*}$ & $1.353-4.129$ \\
\hline Age: & 0.6331 & & \\
\hline $0-19$ & & 0.867 & $0.240-3.133$ \\
\hline $20-30$ & & 1.098 & 0.616-1.959 \\
\hline $40-59$ & & 1.012 & $0.654-1.564$ \\
\hline $80-99$ & & 1.587 & $0.870-2.895$ \\
\hline BMI**: & 0.0713 & & \\
\hline Underweight & & $2.387 *$ & $1.202-4.742$ \\
\hline Overweight & & 0.992 & $0.647-1.521$ \\
\hline Obesity class 1 & & 0.699 & $0.356-1.376$ \\
\hline Obesity class 2 & & 1.571 & $0.657-3.755$ \\
\hline Obesity class 3 & & 1.243 & $0.435-3.552$ \\
\hline Reason for visit: & $0.0007 *$ & & \\
\hline Treatment & & $0.435 * / * * *$ & $0.262-0.720$ \\
\hline Ambulatory follow-up & & $0.312 * / * * *$ & $0.184-0.529$ \\
\hline Results of physical examination & & $0.687 * * *$ & $0.324-1.456$ \\
\hline Course of investigation & & $1.232 * * *$ & $0.531-2.859$ \\
\hline
\end{tabular}

Table 3: The association between "eaten less" and sex, outpatient clinic, age and BMI. Men, hematology department, 60-79 years, normal weight and investigation are the reference group.

$* \mathrm{p} \leq 0.05$ level of significance

** BMI is classified according to the WHO classification of BMI groups

*** Adjusted for sex, BMI, department and age.

There were no association between "eaten less" and age and BMI ( $p>0.05$ ). Only 168 of the total sample had at any time point during disease talked to a professional about weight loss, and only a few were offered intervention.

\section{Discussion}

In the present study, we investigated the prevalence of risk of disease related malnutrition by unintentional weight loss in 713 outpatients from five different outpatient clinics, at Aalborg University Hospital. 
Overall $25.6 \%$ of the consecutive sample, had lost weight unintentionally, and $21 \%$ had eaten less than usual within the past week. Mean weight loss in the overall population was 7.5 Kilos. In most patients, a loss of this amount of weight is quite significant and will be noted by patients and relatives. Since any weight loss, according to former studies is associated with negative outcomes $[5,11,13,25,26]$. Moreover, we found significant risk factors associated to the two outcomes. Underweight and older patients ( $>80$ years) were associated with unintended weight loss $(p<0.05)$ and had a significant higher risk of having an unintended weight loss. Furthermore, women and patients with a BMI $<18.5$ were associated with eaten less within the past week $(\mathrm{p}<0.05)$.

The prevalence of disease related malnutrition is measured by the variables "unintended weight loss" and "eaten less'. These variables have also been used in other studies that have investigated the same problem [27-31] At the same time, the results in the present study showed that $21 \%$ had eaten less, while $26 \%$ had an unintended weight loss. These results are consistent with the results from other studies in France and Switzerland $[28,32]$.

Moreover, the results showed that nausea, pain and no appetite were the most common nutritional impact symptoms in those who suffered unintended weight loss within the past three months and eaten less within the past week. These nutritional impact symptoms have earlier been identified in connection with weight loss during treatment $[33,34]$.

This strengthens the external validity of the present study. Based on the statistical analysis, the reason for visit "Ambulatory follow-up" was a protective factor to having unintended weight loss and eaten less $(\mathrm{p}<0.05)$.

However, another study showed that outpatients found it difficult to maintain weight when switched from treatment to control [35]. This is contradictory to the finding in the present study. In the present study, it is unknown how long time the patients have been in ambulatory follow-up. This may have had an impact on how long they have had to recover from treatment and the opportunity to increase the weight. As the survey is conducted at five outpatient clinics at a Danish hospital and involving over 700 outpatients, it is assumed that the results can be generalized to the same outpatient clinics at other Danish hospitals. The significant amount of weight loss would have been found in professionals if patients were screened in the outpatient care setting, or maybe even earlier in general practice. This amount of weight loss should be seen and addressed by professionals, in order to provide patients with a nutritional care plan, and to give patients the ability to enhance knowledge of risks and possibilities for actions which improve outcomes [14]. A full nutrition screening may not be necessary, since the present study, as well as former studies, have found it feasible to only ask a few questions to patients [24].

Given the results of the present study, asking patients about involuntary weight loss during the past three months, and if they eat less than usual during the past week, may be sufficient to identify nutritional risk in the outpatient setting.

The questionnaire used has some limitations. It is not known how long the patients have been connected to the outpatient clinic, how often they are at the clinic as well as we did not investigate diagnosis or severity of disease. These three elements could be relevant, as there may be differences between the specific disease and treatment and the time when disease related malnutrition occurs $[15,34,36]$.

The questionnaires were collected by different investigators, which may have influenced how the patients have been guided in answering the questionnaire.

\section{Conclusion}

The prevalence of disease related malnutrition is sufficiently measured in outpatient clinics by the variables "unintended weight loss" and "eaten less'.

Based on the statistical analysis, $25.6 \%$ of the outpatients had an unintentional weight loss within the past three months and $21 \%$ had eaten less within the past week.

Underweight and older patients were in particular associated with unintended weight loss and had a significant higher risk of unintended weight loss. Women and underweight were moreover associated with eaten less within the past week. Based on the statistical analysis, there is a high risk of unintended weight loss in outpatient clinics. Therefore, guidelines for handling disease related under nutrition in our patient settings are required.

Conflicts of Interest: The authors have no conflicts of interest to declare for this study.

Acknowledgments: The authors want to express our gratitude to patients, staff and volunteers at the five clinics for their positive attitude and participation towards making this study happen.

\section{Funding: None}

\section{References}

1. Holst M, Mortensen MN, Jacobsen BA, Rasmussen $\mathrm{HH}$. Efficacy of serving bedside in-between meals - 
An intervention study in three medical departments. ESPEN, the European e-Journal of Clinical Nutrition and Metabolism. 2010;5(1):30-36.

2. Ingeman A, et al. Lifestyle Factors and Early Clinical Outcome in Patients with Acute Stroke. Stroke. 2017;48(3):611-617.

3. Saunders J, Smith T. Malnutrition: Causes and consequences. Clin Med J R Coll Physicians London. 2010;10(6):624-627.

4. Norman K, Pichard C, Lochs H, Pirlich M. Prognostic impact of disease-related malnutrition. Clin Nutr. 2008; 27(1):5-15.

5. Sze S, Pellicori P, Zhang J, Clark AL. Malnutrition, congestion and mortality in ambulatory patients with heart failure. Heart. 2018;18. pii: heartjnl-2018-313312.

6. Loh K.W, et al. Unintentional weight loss is the most important indicator of malnutrition among surgical cancer patients. Neth J Med. 2012;70(8):365-369.

7. Bosch $\mathrm{X}$, et al. Unintentional weight loss: Clinical characteristics and outcomes in a prospective cohort of 2677 patients. PLoS One. 2017;12(4):e0175125.

8. Cederholm T, et al. ESPEN guidelines on definitions and terminology of clinical nutrition. Clin Nutr. 2017;36(1):49-64.

9. National Alliance for Infusion Therapy and the American Society for Parenteraland Enteral Nutrition Public Policy Committee and Board of Directors. Disease-related malnutrition and enteral nutrition therapy: a significant problem with a cost-effective solution. Nutr Clin Pract. 2010;25:548-554.

10. Pedersen KM. Health economy by malnutrition among elderly hospitalized patients. Perspekt-Tidsskr om sukker og ernæring. 2009;4(1).

11. Aapro M, et al. Early recognition of malnutrition and cachexia in the cancer patient: A position paper of a European School of Oncology Task Force. Ann Oncol. 2014;25(8):1492-1499.

12. Fearon K, et al. Definition and classification of cancer cachexia: An international consensus. Lancet Oncol. 2011;12(5):489-1495.

13. Martin L, et al. Diagnostic criteria for the classification of cancer-associated weight loss. J Clin Oncol. 2015;33(1):90-99.

14. Holst M, Rasmussen HH. NutriDia-Nutritional Decision Support between Cancer. Ann Clin Case Rep. 2019; 4:1581.

15. Abbott J, Teleni L, McKavanagh D, Watson J, McCarthy A, Isenring E. A novel, automated nutrition screening system as a predictor of nutritional risk in an oncology day treatment unit (ODTU). Support Care Cancer. 2014;22(8):2107-2112.
16. Collins PF, Stratton RJ, Elia M. Nutritional support in chronic obstructive pulmonary disease: a systematic review and meta-analysis. Am J Clin Nutr. 2012;95(3):1385-1395.

17. Collins PF, Elia M, Stratton RJ. Nutritional support and functional capacity in chronic obstructive pulmonary disease: A systematic review and metaanalysis. Respirology. 2013;18(4):616-29.

18. Weekes CE, et al. Oral nutrition supplements and between-meal snacks for nutrition therapy in patients with COPD identified as at nutritional risk: a randomised feasibility trial. BMJ Open Respir Res. 2019;6(1): e000349.

19. Baldwin C, Spiro A, Ahern R, Emery PW. Oral nutritional interventions in malnourished patients with cancer: A systematic review and meta-analysis. J Natl Cancer Inst. 2012;104(5):371-385.

20. Lagiu JA, et al. Prediction model to predict critical weight loss in patients with head and neck cancer during (chemo)radiotherapy. Oral Oncol. 2016;52(2016):91-96.

21. Danish national Board of Health. A Guide for Doctors, Nurses, Healthcare Assistants, Auxiliary Nurses and Screening and Clinical Dietitians,Screening and treatment of patients at nutritional risk. 2008.

22. McGurk P, Jackson JM, Elia M. Rapid and reliable self-screening for nutritional risk in hospital outpatients using an electronic system. Nutrition. 2013;29(4):693-6.

23. Álvaro Sanz E, et al. Nutritional risk and malnutrition rates at diagnosis of cancer in patients treated in outpatient settings: Early intervention protocol. Nutrition. 2019;57:148-153.

24. Tangvik R, et al. The nutritional strategy: Four questions predict morbidity, mortality and health care costs. Clin Nutr. 2013;33(4):634-41.

25. Yaxley A, Miller MD, Fraser RJ, Cobiac L, Crotty M. The complexity of treating wasting in ambulatory rehabilitation: Is it starvation, sarcopenia, cachexia or a combination of these conditions? Asia Pac J Clin Nutr.

2012;21(3):386-93.

26. Yaxley A, Crotty M, Miller M. Identifying Malnutrition in an Elderly Ambulatory Rehabilitation Population: Agreement between Mini Nutritional Assessment and Validated Screening Tools. Healthcare (Basel). 2015;3(3):822-829. 
27. Andersson J, Hulander E, Rothenberg E, Iversen PO. Effect on body weight, quality of life and appetite following individualized, nutritional counselling to homeliving elderly after rehabilitation - An open randomized trial. J Nutr Heal Aging. 2017;21(7):811-818.

28. Uster A, et al. Influence of a nutritional intervention on dietary intake and quality of life in cancer patients: A randomized controlled trial. Nutrition. 2013;29(1112):1342-1349.

29. Starke J, Schneider H, Alteheld B, Stehle P, Meier R. Short-term individual nutritional care as part of routine clinical setting improves outcome and quality of life in malnourished medical patients. Clin Nutr. 2011;30(2):194-201.

30. van den Berg MG, et al. Comparison of the effect of individual dietary counselling and of standard nutritional care on weight loss in patients with head and neck cancer undergoing radiotherapy. Br J Nutr. 2010 Sep;104(6):872877.

31. Eglseer D, Schoberer D, Halfens R LC. The impact of using a malnutrition screening tool in a hospital setting: a mixed methods study. Eur J Clin Nutr. 2019;73(2):284-92.

32. Hébuterne X, Lemarié E, Michallet M, de Montreuil CB, Schneider SM GF. Prevalence of malnutrition and current use of nutrition support in patients with cancer. JPEN J Parenter Enteral Nutr. 2014;38(2):196-204.

33. Abbott J, Teleni L, McKavanagh D, Watson J, McCarthy AL, Isenring E. Patient-Generated Subjective Global Assessment Short Form (PG-SGA SF) is a valid screening tool in chemotherapy outpatients. Support Care Cancer. 2016;24(9):3883-3887.

34. Isenring E, Cross G, Kellett E, Koczwara B DL. Nutritional status and information needs of medical oncology patients receiving treatment at an Australian public hospital. Nutr Cancer. 2010;62(2):220-228.

35. Poulsen, Grith M. Østerlind K, Andersen JR, Pedersen LL, Bæksgaard L. Randomized trial of the effects of individual nutritional counseling in cancer patients. Clin Nutr. 2013;33(5):749-753.

36. Khalid U, et al. Symptoms and weight loss in patients with gastrointestinal and lung cancer at presentation. Support Care Cancer. 2007;15:39-46. 